\title{
L'approche Par Competences Et La Question De La Montee Des Inegalites Scolaires En Cote d'Ivoire. Analyse Des Pratiques Des Pratiques Enseignantes En Classe Sur Le Profil De Sortie Des Eleves Du Premier Cycle Du Secondaire En Cote d'Ivoire.
}

\author{
Dr. Nguessan Kouamé, (Maître de Conférences) \\ Ecole Normale Supérieure d'Abidjan/ \\ Département des Sciences de l'Education, Côte d'Ivoire
}

Doi:10.19044/esj.2018.v14n34p307 URL:http://dx.doi.org/10.19044/esj.2018.v14n34p307

\begin{abstract}
Considered as a tool to understand the academic achievements, the orientations, the access to the different sectors, the obtaining of diplomas, etc., the concept of school inequalities is the prism by which we evaluate the school, its operation and its ability to fulfill the explicit missions that are its own. We specifically question the impact of classroom teaching practices on the exit profile of students in the "Science" field and consequently on the graduation rate of students in the secondary education of the first cycle, which is a necessary condition of access. to have a job; and therefore a factor of school inequalities.

This study aims to point out certain difficulties and obstacles observed in reading the didactic intentions of the educational programs by the teachers. Surveys based on observation grids on the one hand, audio and video recordings, and interviews on the other, were conducted on three types of teachers (competent, efficient, and expert) by discipline in each of the three disciplines of the field "Science" in the fourth year of junior high school. The results show that these teachers, despite their certain skills, have many shortcomings in mastering the components of the different knowledge required for effective teaching / learning. As a result, some teaching strategies have to be adjusted to fill the knowledge gap among the latter. Given these shortcomings, continuous teacher training that takes into account all disciplinary, curricular, didactic and pedagogical aspects is necessary to give the learner the desire to follow, to pursue studies, develop more skills, and consequently reduce school inequalities.
\end{abstract}


Keywords: School inequalities, Teachers 'professional knowledge, Middle school, Skills approach, Exit profile

\section{Résumé}

Considéré comme un outil pour comprendre les acquis scolaires, les orientations, l'accès aux différentes filières, l'obtention de diplômes, etc., le concept d'inégalités scolaires est le prisme par lequel on peut évaluer l'école, son fonctionnement et sa capacité à remplir les missions explicites qui sont les siennes. Nous interrogeons spécifiquement l'impact des pratiques enseignantes en classe sur le profil de sortie des élèves du domaine « Sciences » et par conséquent sur le taux d'obtention des élèves du diplôme du premier cycle de l'enseignement secondaire, condition nécessaire d'accès à l'emploi ; et donc un facteur d'inégalités scolaires.

Cette étude vise à pointer certaines difficultés et obstacles constatés dans la lecture des intentions didactiques des programmes éducatifs par les enseignant(e)s. Des enquêtes basées sur des grilles d'observations d'une part, des enregistrements audio et vidéo, et des entretiens d'autre part, ont été menées sur trois types d'enseignant(e)s (compétent, efficace, et expert) par discipline dans chacune des trois disciplines du domaine "Sciences » en classe de quatrième année de collège. Il ressort des résultats que ces enseignant(e)s malgré leurs compétences certaines, présentent de nombreuses lacunes dans la maîtrise des composantes des différentes connaissances nécessaires à un enseignement/apprentissage efficace. Par conséquent, certaines stratégies d'enseignement sont à ajuster pour combler le déficit de connaissances chez ces derniers. Au regard de ces insuffisances, une formation continue des enseignant(e)s qui prend en compte tous les aspects disciplinaire, curriculaire, didactique, et pédagogique est nécessaire pour donner à l'apprenant(e) l'envie de suivre, de poursuivre les études, de développer plus de compétences, et réduire conséquemment les inégalités scolaires.

Mots-clés : Inégalités scolaires, Connaissances professionnelles des enseignants, premier cycle du secondaire, Approche par compétences, profil de sortie.

\section{Introduction:}

Considéré comme un outil pour comprendre les acquis scolaires, les orientations, l'accès aux différentes filières, l'obtention de diplômes, etc., le concept d'inégalités scolaires est le prisme par lequel on peut évaluer l'école, son fonctionnement et sa capacité à remplir les missions explicites qui sont les siennes.

L'enjeu majeur de l'école est d'outiller les élèves en instruments et méthodes nécessaires, afin qu'ils puissent inférer les compétences au terme de 
leur cycle scolaire. Les nombreuses réformes des programmes d'enseignement et les politiques mises en œuvre sont non seulement de répondre efficacement aux exigences du monde moderne mais aussi et surtout de rendre les apprenants autonomes, faciliter leur intégration dans le contexte mondial, et de lutter contre l'échec scolaire dont les effets majeurs sont les obstacles cognitifs et les difficultés à utiliser les acquis en situations complexes.

Le diagnostic du système éducatif ivoirien (RESEN, 2011) révèle que les élèves après le premier cycle de l'enseignement secondaire éprouvent d'énormes difficultés aussi bien au niveau de l'acquisition des connaissances que dans le développement des compétences, et ils maintiennent un taux d'obtention de diplômes inférieur. Le Rapport UNESCO fonds Chinois pour la Côte d'Ivoire (2013) relève que de 2005 à 2010, le taux moyen d'obtention de diplômes en fin de cycle est de $30 \%$; et qui chute à $17 \%$ pour les années 2011 et 2012. C'est pourquoi ces derniers ont fait l'objet d'une attention particulière afin de contrer le décrochage scolaire qui les menace. Notamment, par l'évaluation de la nouvelle approche en vigueur, l'approche par compétences introduite pour la première fois dans le système éducatif ivoirien en 2002, et rentrée en vigueur dans l'enseignement secondaire pendant l'année scolaire 2012/2013 en vue de prévenir ce décrochage. Il s'agit d'impliquer désormais les élèves dans le travail mené en classe, de susciter leur intérêt et d'obtenir leur adhésion sincère à la construction du savoir. Or, cette nouvelle approche mise en place dans l'enseignement secondaire en Côte d'ivoire se heurte à certaines difficultés et produit peu de résultats positifs sur le taux d'obtention de diplômes en fin du premier cycle.

Par ailleurs, il a été établi que ces élèves qui arrivent en classe de seconde présentent de nombreuses conceptions erronées dans la plupart des disciplines, lesquelles sont accentuées par des attitudes négatives véhiculées face à ces disciplines. En fait, il est possible de distinguer dans ce groupe d'élèves un cumul de retard après la classe de troisième ( $4^{\text {ème }}$ année du premier cycle de l'enseignement secondaire).

Puisque ces retards scolaires cumulés au niveau de la fin du premier cycle de l'enseignement secondaire, semblent avoir un impact important sur le décrochage au niveau secondaire, il devient alors essentiel de privilégier des interventions dans les pratiques pédagogiques pour améliorer la performance et augmenter les probabilités d'obtention du diplôme en fin de ce cycle. C'est l'objectif poursuivi par cette recherche. Plus précisément, il s'agit de mesurer l'impact des pratiques enseignantes en classe sur le profil de sortie des élèves et par conséquent sur le taux d'obtention des diplômes après l'adoption de l'approche de compétences.

Ainsi, l'article s'organise de la manière suivante. Dans une première partie, nous présentons le cadre théorique et la problématique ; ce qui permet de construire les questions de recherche. Une deuxième partie traite de la 
méthodologie de nos travaux. Les résultats pour mesurer les inégalités scolaires sont décrits dans une troisième partie. La dernière partie explicite les causes et en discute leur portée.

\section{Cadre théorique}

\section{Approche par compétences (APC) et profil de sortie}

Selon Masciotra et Medzo (2009) ; Jonnaret et Masciotra (2004), l'approche par compétences est basée sur une épistémologie constructiviste et socioconstructiviste. Cela est appuyé par les travaux de Jonnaert et al. (2005) où ils indiquent que l'approche constructiviste vise la construction des connaissances et le développement des compétences par un apprenant actif. Cependant comme le fait observer Jonnaert (2007), les programmes d'études continuent d'être développés selon une organisation des contenus d'apprentissage par objectifs et la transmission de savoirs. Pour éviter un tel glissement épistémologique, dans leurs travaux, Jonnaert et al. (2005), puis Jonnaert (2007) proposent l'approche située de la compétence pour concevoir les programmes d'études. Ainsi, dans une approche située, la personne, ses actions et les circonstances forment un tout qui ne peut être séparé ; et dans la conception de l'apprentissage, la compétence se développe « en action et en situation » et n'est constatée que lorsque le traitement de la situation est réussi et acceptable. En d'autres termes, une compétence se comprend dans l'agir d'une personne qui solutionne avec succès une situation (Masciotra et Medzo, 2009). Contrairement aux approches de formation par compétences qui proposent un profil de sortie décrivant des connaissances, des habiletés et des attitudes essentielles en regard d'objectifs ou de compétences à atteindre, les programmes situés prônent une entrée par les situations de vie (Jonnaert et al., 2005).

Selon Otis et Ouellet (1996), le profil de sortie est un ensemble intégré de connaissances, d'habiletés et d'attitudes attendu au terme de la formation et qui permet de guider et d'orienter le travail éducatif dans le programme d'études. Toutes les habiletés, les attitudes et les connaissances identifiées préalablement devraient pouvoir être associées à une compétence. Il est important de s'assurer que toutes et tous les utilisateurs ont la même compréhension des termes employés pour définir les compétences, les habiletés, les attitudes et les connaissances.

Pour Masciotra et Medzo (2009), le profil de sortie prescrit des classes de situations extraites du contexte de la vie personnelle des élèves par la formation. Un profil de sortie développé à partir de situations de classes vécues par l'ensemble des élèves pourrait répondre aux besoins réels de formation. Le traitement de ces classes de situations en contexte de formation leur permettrait de développer les compétences nécessaires pour s'adapter aux situations qu'ils rencontrent au quotidien. 
Ainsi, dans le processus Enseignement/Apprentissage, l'approche par compétences (Roegiers, 2007) permet à l'élève d'acquérir des compétences durables susceptibles de l'aider dans son parcours éducatif et dans la vie quotidienne. Elle met l'accent sur tout ce qui est fondamental afin de garantir une meilleure transmission des savoirs. L'approche par compétences devient donc la base pédagogique de tous les constituants de l'enseignement. Les actions et les réflexes de l'apprenant deviennent la principale source de son apprentissage, elle vise à mettre l'apprenant dans le centre du processus éducatif pour lutter contre son échec.

Le profil de sortie de l'éducation de base du domaine « sciences » du premier cycle de l'enseignement secondaire en vigueur actuellement en Côte d'Ivoire se présente comme indique le tableau 1 ci-dessous.

Tableau 1 : caractéristiques du profil de sortie pour chacune des disciplines du domaine "Sciences"

\begin{tabular}{|c|c|c|}
\hline Domaine & Disciplines & Profil de sortie \\
\hline & Mathématiques & $\begin{array}{l}\text { A la fin du premier cycle, l'élève doit avoir acquis des connaissances et des } \\
\text { compétences lui permettant de traiter des situations relatives : } \\
\text { - aux configurations du plan : point, droite, demi-droite, segment, } \\
\text { triangle, angle, cercle, parallélogramme, vecteurs ; } \\
\text { aux transformations du plan : symétrie centrale, symétrie } \\
\text { orthogonale, translation ; } \\
\text { aux configurations de l'espace : pavé droit, cylindre droit, } \\
\text { prisme droit, pyramide régulière, cône de révolution et leur } \\
\text { représentation en perspective cavalière ; } \\
\text { aux activités numériques : nombres réels (calculs dans } \mathbf{N}, \mathbf{Z}, \mathbf{D} \text {, } \\
\text { Q et } \mathbf{R}) \text {, calcul littéral (factorisation, développement, réduction } \\
\text { et simplification d'une expression littérale, équations, } \\
\text { inéquations); } \\
\text { à l'organisation des données : proportionnalité et statistique ; } \\
\text { à la géométrie analytique : Coordonnées d'un vecteur, } \\
\text { Equations de droites. }\end{array}$ \\
\hline & Physique Chimie & $\begin{array}{l}\text { A la fin du premier cycle, l'élève doit avoir acquis des connaissances et des } \\
\text { compétences lui permettant de traiter des situations se rapportant : } \\
\text { - } \quad \text { aux lentilles et aux défauts de l'œil ; } \\
\text { - } \quad \text { aux forces, à la masse volumique, à l'énergie et à la puissance } \\
\text { - et à la puissance mécaniques ; } \\
\text { - aux lois des intensités et des tensions, à la loi d'Ohm, à la } \\
\text { puissance et à l'énergie électriques ; } \\
\text { - } \quad \text { aux réactions chimiques et aux solutions aqueuses. }\end{array}$ \\
\hline 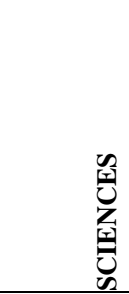 & $\begin{array}{l}\text { Sciences de la Vie et de } \\
\text { la Terre }\end{array}$ & $\begin{array}{l}\text { A la fin du premier cycle, l'élève doit avoir acquis des connaissances et des } \\
\text { compétences lui permettant de traiter des situations liées : } \\
\text { - aux fonctions vitales (nutrition, reproduction, relation) chez } \\
\text { - l'homme, les animaux et les végétaux; } \\
\text { aux manifestations géologiques et leur impact sur la qualité de } \\
\text { la vie ; } \\
\text { - aux phénomènes liés à la pédagogie, à l'environnement et à la } \\
\text { - santé ; } \\
\text { et de développer un raisonnement scientifique. }\end{array}$ \\
\hline
\end{tabular}

Source : Cadre d'Orientation et du Curriculum, $2017^{14}$

${ }^{14}$ Concernant le rendement interne du système éducatif, le taux de redoublement est de $18 \%$ dans le premier cycle secondaire et $19 \%$ dans le second cycle en 2007. Ces taux qui restent élevés, réduisent les capacités d'accueil des infrastructures scolaires et influencent 


\section{Les inégalités scolaires et qualité de l'éducation}

Pour Felouzis (2012), le concept d'inégalités scolaires est un outil pour comprendre comment et pourquoi les acquis scolaires, les orientations, l'accès aux différentes filières, l'obtention de diplômes, etc., sont statistiquement liés aux caractéristiques des individus : leur origine sociale et leur niveau socioéconomique, leur sexe ou leur origine culturelle. Selon Felouzis et al. (2016), c'est le caractère collectif dans le moindre accès à un bien éducatif (ressources d'enseignement, diplôme...) qui constitue une inégalité scolaire, par exemple lorsqu'un bien est moins distribué dans une catégorie sociale particulière (par exemple, les enfants d'ouvriers, certaines communautés issues de l'immigration, les filles (vs les garçons)). C'est également l'ampleur des disparités entre individus qui fonde l'existence des inégalités sociales à l'école. Cependant, pour ces auteurs, lorsqu'apparaissent des inégalités relatives à des groupes d'individus, on s'écarte du registre du mérite individuel, et une intervention publique à fonction corrective est nécessaire. Ils insistent donc sur la nécessité de prendre en compte le caractère collectif des inégalités. Leur ampleur et leur évolution spatio-temporelle doivent aussi être prises en compte, pour juger de la légitimité d'une inégalité.

Les travaux de Coleman et al. (1966) et de Jencks (1992) revèlent que la grande variabilité des conditions d'apprentissage était un facteur fortement explicatif des inégalités scolaires. Les discriminations négatives notées par Crahay et al. (2013) dont certains élèves et groupes d'élèves sont l'objet, sont le fruit d'une variation de la qualité de l'enseignement liée à des facteurs institutionnels tels que le financement des écoles, les modes de recrutement d'enseignants plus ou moins expérimentés (du débutant à l'expert) ou encore la ségrégation scolaire liée aux modes d'affectation des élèves dans les établissements.

Les travaux de Wang et al. (1990) sur plusieurs ouvrages scolaires mentionnent que les programmes enseignés et les caractéristiques contextuelles telles que la nature du groupe de référence, la qualité et la quantité d'instruction effectivement reçue par les élèves, constituent un facteur déterminant du niveau d'apprentissage. Et donc, la gestion de la classe et du climat d'une part ; la nature des interactions entre élèves et professeurs d'autre part, représentent une constellation importante de variables liées à un enseignement efficace (Wang et al. 1990).

négativement l'efficacité interne et l'efficience du système éducatif. Les effectifs des enseignants demeurent toutefois insuffisants pour faire face au besoin d'expansion de cet ordre d'enseignement, notamment dans les disciplines scientifiques et en français. Par ailleurs, la répartition spatiale du personnel enseignant dans le secondaire révèle de fortes disparités, avec une concentration dans les grands centres urbains. Les taux élevés de redoublement et les faibles taux d'achèvement, attestent des insuffisances de l'efficacité et de l'efficience du système. 
Selon Rochex (2008), le processus de production des inégalités scolaires est accentué par les évolutions des pratiques pédagogiques qui placent de plus en plus l'activité des élèves en classe au principe des apprentissages. Ainsi, à partir de nombreuses observations de terrain dans les classes en France, il relève que dans la plupart de ses observations, il s'est avéré que l'intention d'enseigner du professeur ne soit pas (plus) déterminée en termes de savoirs, mais en terme, de situations et de tâches. L'effectuation et l'enchaînement des tâches à exécuter sont censés conduire par eux-mêmes à un apprentissage, ou du moins à des savoir-faire que les élèves devraient être à même de mobiliser dans d'autres situations, d'autres tâches.

Il conclut donc que ce type d'ambiguïté crée les conditions d'inégalités scolaires au sens où elles nécessitent un décodage par les élèves et leur famille de façon à identifier, sous les tâches à accomplir, les savoirs scolaires à acquérir. Les processus de production des inégalités scolaires se fondent donc sur les pratiques pédagogiques dont la « transparence » et les buts apparaissent comme plus ou moins explicites aux élèves en fonction de leur capital culturel.

De l'ensemble des travaux ci-dessus mentionnés, nous notons que les sources d'analyse des inégalités scolaires sont aujourd'hui multiples et empruntent à différentes traditions de recherche. Toutes les différences entre les élèves ne constituent pas des inégalités scolaires illégitimes.

\section{Connaissances professionnelles des enseignants (PCK)}

Il s'agit des connaissances mobilisées par l'enseignant dans son enseignement de sa discipline au collège. Cela nécessite de prendre en compte l'activité de ce dernier dans l'interactivité enseignant-apprenant où certains types de connaissances sont construites pendant cette interactivité. Cette interaction reconnue comme modèle de Shulman (1986), concerne d'un côté, le savoir et sa transmission et, de l'autre côté, l'activité des enseignants en contexte de travail.

Dans notre étude, ce modèle proposé par Shulman $(1986,1987)$ et modifié par Grossman (1990) et Magnusson et al. (1999) est précieux pour reconstruire les connaissances en jeu dans la pratique de l'enseignant. Il permet de distinguer les connaissances qui se situent au niveau enseignant, celles qu'il mobilise en lien avec un contenu à enseigner, des connaissances au niveau élève qui sont les connaissances spécifiques à l'enseignement de ce contenu. Les connaissances pédagogiques liées au contenu (Pedagogical Content Knowledge (PCK)) expriment la combinaison (l'amalgame) du contenu et de la pédagogie. L'étude de Grossman (1990) définit les quatre grands domaines de connaissance de l'enseignant qui sont : les connaissances pédagogiques générales (PK), les connaissances disciplinaires (SMK), les connaissances pédagogiques liées au contenu disciplinaire (PCK), et les connaissances du contexte (KofC). Mais ce travail de reconstruction de 
connaissances à partir de l'action de l'enseignant dans la classe est difficile car, selon Vergnaud (1996), c'est la forme opératoire de la connaissance qui est utilisée dans l'action. Or, elle est en décalage avec la forme prédicative, ce qui signifie qu'il existe des connaissances implicites, qui sont difficilement identifiables. Ainsi, Magnusson et al. (1999) définissent un modèle des PCK suivant cinq composantes :

- Connaissances sur l'orientation pour l'enseignement de la discipline : elles se réfèrent aux connaissances des enseignants à propos des buts et des objectifs pour enseigner la discipline à un niveau d'étude particulier ;

- Connaissances des programmes de la discipline : elles concernent la connaissance des buts et objectifs (attentes nationales) et la connaissance spécifique du programme et du matériel pédagogique ;

- Connaissances sur l'évaluation : ce qu'il faut évaluer et comment l'évaluer ;

- Connaissances des stratégies d'enseignement : elles incluent des stratégies spécifiques à l'enseignement de certains sujets. Par exemple, sur un cycle d'apprentissages, l'utilisation d'analogies, de démonstrations ou d'expériences ;

- Connaissances sur les élèves : elles portent sur la connaissance que les enseignants ont des apprentissages des élèves de la discipline : les exigences de l'apprentissage de certaines notions, les parties que les élèves trouvent difficiles, les approches de l'apprentissage de la discipline, et les conceptions alternatives.

Notons que la composante «orientations pour l'enseignement » chapeaute les autres. Cependant, son inclusion dans le modèle des PCK est questionnée pour deux raisons (Abell, 2007). Premièrement, une orientation est théorisée comme une vue générale de l'enseignement de la discipline, et non pas comme des connaissances spécifiques d'un sujet. Deuxièmement, ces vues générales de l'enseignement de la discipline et son apprentissage sont souvent étudiées en tant qu'interaction entre les connaissances, les croyances et les valeurs, et non pas strictement comme une structure de connaissances.

\section{Questions de recherche}

Compte tenu des idées avancées ci-dessus, nous articulons notre travail autour des questions de recherche suivantes :

Les pratiques d'enseignement des enseignants constituent-elles une source d'inégalités scolaires en Côte d'Ivoire ? Autrement dit, les profils de sortie ainsi définis, permettent-ils de réduire les inégalités scolaires, d'accroitre la performance des élèves et augmenter les probabilités d'obtention du diplôme de fin du premier cycle de l'enseignement secondaire? 
Pour répondre à ces interrogations, nous avons suivi 3 types enseignants (compétent, efficace, et expert) dans chacune des trois disciplines du domaine « Sciences » dans la classe, soit un total de 9 enseignants suivis et observés.

\section{Méthodologie}

Nous explicitons, dans ce paragraphe, les outils de recueil et de traitement des données par rapport au contexte de notre étude.

\section{Contexte de l'étude}

Nous avons mis en œuvre cette méthodologie aux premier et deuxième trimestres de l'année scolaire 2017/2018, pour le suivi de trois types d'enseignants (compétent ${ }^{15}$, efficace ${ }^{16}$ et expert ${ }^{17}$ ) par discipline dans chacune des trois disciplines du domaine «Sciences »: Mathématiques, Physiquechimie, Sciences de la Vie et de la Terre. Généralement, ce sont ces trois types d'enseignants qui sont affectés aux élèves des classes de la $3^{\text {ème }}$. L'étude que nous présentons ici se réfère aux leçons suivantes : Les forces en Physiquechimie, Le rôle des aliments simples en Sciences de la Vie et de la Terre, Equations et inéquations dans $\mathbf{R}$ en Mathématiques dans les programmes de la classe de $3^{\text {ème }}$ en vigueur depuis 2015 . Ce choix permet de suivre, chaque enseignant dans la conduite de sa classe. Nous observons les pratiques dans la classe, et les routines installées qui rendraient visibles les évolutions qui nous intéressent dans le suivi des enseignants.

\section{Population et échantillon d'étude}

La population visée par cette étude est celle des enseignants de la classe de $3^{\text {ème }}$ ( $4^{\text {ème }}$ année d'étude au collège). Les classes concernées sont mixtes : généralement $45 \%$ de filles et $55 \%$ de garçons. L'échantillon est composé de 9 enseignants qui ont été invités à prendre part à l'étude de façon volontaire. Il s'agit donc d'un échantillon de convenance, numériquement restreint. Pour une meilleure représentativité, ces enseignants ont été choisis à partir de leur efficacité dans la classe ; c'est-à-dire de leurs caractéristiques personnelles innées et des compétences acquises au cours de leur carrière professionnelle.

\footnotetext{
${ }^{15}$ L'enseignant compétent ( $3^{\text {ème }}$ ou $4{ }^{\text {ème }}$ année d'enseignement) choisit consciemment ce qu'il faut faire et peut isoler les événements clés dans la conduite de sa classe.

${ }^{16}$ L'enseignant efficace (quelques-uns avec cinq ans de pratique et d'autres avec plus d'ancienneté) a construit un large éventail d'expériences et de moyens.

${ }^{17}$ L'enseignant expert (seuls quelques-uns atteignent ce sommet) possède un sens global de la situation, de sorte qu'il y répond avec fluidité, sans même devoir y penser.
} 


\section{Outils de recueil de données}

Le corpus que nous recueillons comporte des enregistrements audio et vidéo de séquences de classe et des entretiens avec chacun des enseignants, des données issues d'une grille d'observation. Cette grille prend en compte les caractéristiques des situations d'apprentissage exploitées pendant les séances d'observation et les différentes connaissances dont a besoin un enseignant pour faire son cours dans la classe, ainsi que la trace écrite de l'enseignant pour analyse en vue de compléter les observations notées récupérée après le cours permettant d'approcher le travail hors classe des enseignants. La grille d'observation en classe est validée par un enseignant-chercheur en didactique des disciplines, un inspecteur pédagogique de la discipline et un conseiller pédagogique dans la discipline. La durée totale d'enregistrement vidéo est celle de la durée du cours. Nous avions deux caméras à disposition : la première était fixe au fond de la classe centrée sur le tableau et la seconde était mobile prenant les interactions entre le professeur et les élèves. L'enseignant était équipé d'un micro-cravate et quatre micros « d'ambiance » étaient placés dans les quatre coins de la classe. Cela permet d'approcher le travail hors classe des enseignants. Après la séance, l'équipe s'entretient avec l'enseignant afin de l'amener à préciser sa pensée pour ajuster certaines incompréhensions. C'est un outil essentiel de la méthodologie d'investigation réflexive dans le sens où il incite à la réflexivité sur les activités (Gueudet \& Trouche, 2010). Le remplissage de la grille d'observation consiste à évaluer la prestation de l'enseignant au regard des composantes des situations d'apprentissage et des types de connaissances. Nous retenons deux critères se rapportant à l'observation d'une séance de classe, à savoir la démarche scientifique et l'argumentation, et qui pourraient être évaluées au moyen d'une même échelle d'excellence comprenant, les trois niveaux « médiocre», «acceptable» et «excellent» (tableau 2). Nous notons dans la grille d'observation, le signe (+) pour le niveau « excellent» dans la prestation de l'enseignant si ce dernier maîtrise la composante de l'objet observé ; le signe (+/-), si on constate des difficultés dans l'explication de l'enseignant ; le signe (-), si l'enseignant a une conception erronée concernant 1'objet de savoir.

Tableau 2 : caractéristiques de chaque critère pour les trois niveaux d'excellence.

\begin{tabular}{|c|c|c|c|}
\hline Critères & Niveau « excellent » & Niveau « acceptable » & Niveau « médiocre » \\
\hline $\begin{array}{l}\text { Démarche } \\
\text { scientifique }\end{array}$ & $\begin{array}{l}\text { L'enseignant introduit des } \\
\text { variables et les utilise à bon } \\
\text { escient }\end{array}$ & $\begin{array}{l}\text { L'enseignant introduit des } \\
\text { variables mais ne les exploite } \\
\text { pas adéquatement }\end{array}$ & $\begin{array}{l}\text { L'enseignant utilise } \\
\text { uniquement des } \\
\text { descriptions textuelles des } \\
\text { grandeurs et n'introduit } \\
\text { pas des variables }\end{array}$ \\
\hline $\begin{array}{l}\text { Argumentatio } \\
\mathrm{n}\end{array}$ & $\begin{array}{l}\text { L'enseignant explique et } \\
\text { justifie toutes les étapes de sa } \\
\text { démarche d'une manière } \\
\text { claire et compréhensible }\end{array}$ & $\begin{array}{l}\text { L'enseignant explique et justifie } \\
\text { maladroitement les grandes } \\
\text { lignes de sa démarche }\end{array}$ & $\begin{array}{l}\text { L'enseignant enchaîne des } \\
\text { étapes sans explication ni } \\
\text { justification }\end{array}$ \\
\hline
\end{tabular}




\section{Analyse des données}

Nous analysons le degré de maîtrise des connaissances et des pratiques de classes des enseignants qui caractérisent l'approche par compétences afin d'évaluer leur effet sur le taux de réussite des élèves de la classe de $3^{\text {ème }}$. Nous avons donc utilisé des données provenant des grilles d'observation, des enregistrements et les entrevues individuelles. L'analyse de contenu s'est effectuée sur les catégories de connaissances et sur celles relatives aux pratiques de classe. Les objets d'observation examinés sont les suivants : les situations d'apprentissage, les connaissances de la discipline, les connaissances curriculaires, les connaissances pédagogiques, les connaissances didactiques, et les connaissances des TICE. Les analyses plus fines sont faites sur les composantes des différents objets examinés.

\section{Résultats et discussion}

Pour répondre à nos questions de recherche, l'ensemble des données recueillies ont été traitées en fonction des composantes des six objets d'observation. Notre but est de rapporter pour chaque type d'enseignant la maîtrise de chacune des composantes des différents objets d'observation pendant sa prestation dans la classe. Ces résultats vont nous permettre d'évaluer le degré de maîtrise de l'approche par compétences et ses conséquences sur la réussite des élèves.

\section{- Premier objet d'observation : les situations d'apprentissage}

Dans la classe, la situation d'apprentissage s'organise en trois étapes : d'abord, la préparation à l'apprentissage où l'enseignant(e) transmet l'information nécessaire à l'élève pour qu'il soit prêt pour la tâche ; ensuite, la réalisation de la tâche où l'enseignant(e) modélise la tâche et guide l'élève dans la façon de faire la tâche et de traiter l'information reçue; enfin, le transfert de l'apprentissage dans d'autres situations où l'enseignant(e) fournit d'autres situations d'apprentissage et l'élève réinvestit ce qu'il a appris.

Le tableau 3 répertorie les évaluations relevées sur les observations classe de 9 enseignants dans l'utilisation des situations d'apprentissage pendant leur séance de classe. 
Tableau 3 : synthèse des observations au regard des situations d'apprentissage pour les trois profils d'enseignants choisis

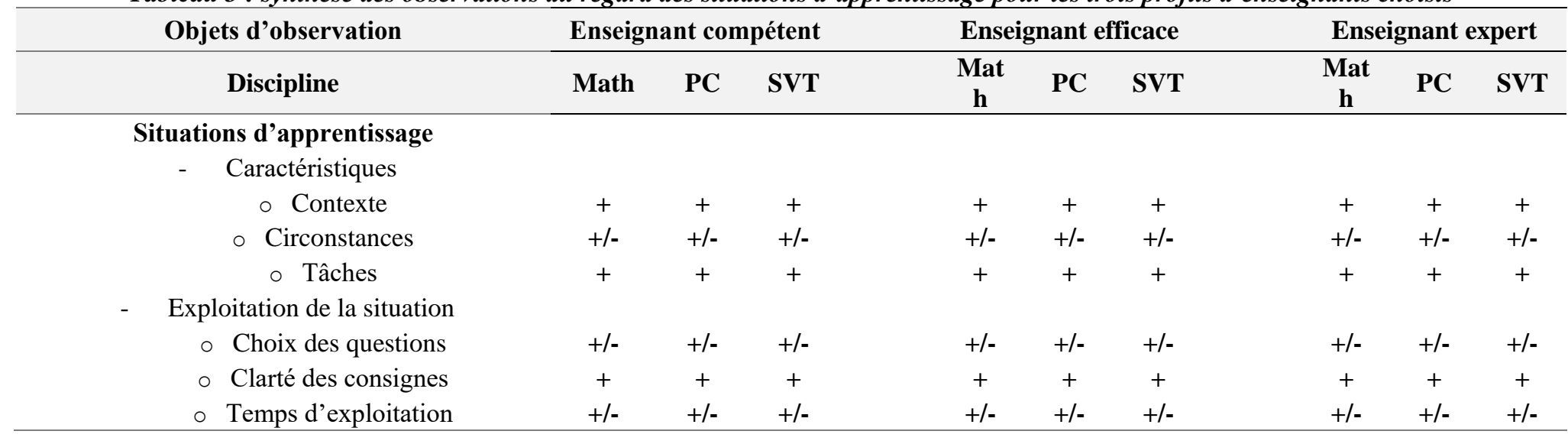


Notre jugement sur la prestation de chacun des trois profils d'enseignants retenus au regard des situations d'apprentissage, indique que les 9 enseignants observés ont une parfaite maitrise du contexte et des tâches des situations d'apprentissage exploitées. Concernant la clarté de la question, nous notons les enseignants la réussissent parfaitement. Cependant, nous notons une défaillance de la prise en compte des circonstances dans le texte de la situation d'apprentissage. En effet, nous relevons que la plupart des textes proposés ne permet pas aux apprenants d'identifier clairement cette partie. Quant à l'exploitation de la situation, nous constatons que les enseignants compétents et efficaces éprouvent des difficultés. Ces difficultés sont aussi observées au niveau du temps d'exploitation où cette fois-ci, les enseignants observés vont au-delà du temps alloué. Cet ensemble de difficultés constitue une dérive pédagogique qui entrave l'articulation effective entre les trois parties de la situation d'apprentissage. Cet écart nous montre évidemment que malgré les impressions positives constatées au niveau de certaines composantes de la situation d'apprentissage, il a encore une marge d'amélioration. Si nous convenons que l'exploitation de la situation repose sur le principe suivant: «l'esprit de celui qui apprend procède non pas du simple au complexe mais d'une complexité implicite et diffuse vers une complexité explicite et distincte », alors elle met en compétition les compétences, les acquis, le rythme, et le profil pédagogique de l'enseignant. Ainsi, maitriser la conception des situations d'apprentissage, c'est être capable de mobiliser ses acquis dans des tâches et des situations complexes, à l'école puis dans sa vie d'adulte (Scallon, 2004).

\section{- Deuxième objet d'observation : les connaissances de la discipline}

Ce sont les connaissances nécessaires pour enseigner la discipline et qui font référence aux connaissances que l'enseignant doit posséder relativement aux concepts et aux procédures de la discipline, aux stratégies de résolution des problèmes de même qu'aux liens qui existent entre ses différentes composantes. Les connaissances du contenu disciplinaire sont les connaissances que l'enseignant est chargé de construire avec les élèves, mais ces connaissances ne se limitent pas à celles qu'il doit enseigner. Les connaissances du contenu disciplinaire peuvent être de deux natures : elles peuvent porter sur l'organisation des concepts, faits, principes et théories ou bien sur les règles régissant les preuves utilisées pour généraliser et justifier les connaissances produites par la discipline (Schwab, cité dans Abell, 2007). Le tableau 4 répertorie les évaluations relevées sur les observations de classe des trois profils sur les connaissances de la discipline. 
Tableau 4 : synthèse des observations au regard des connaissances de chacune des disciplines pour les trois profils d'enseignants choisis

\begin{tabular}{|c|c|c|c|c|c|c|c|c|c|}
\hline \multirow{2}{*}{$\begin{array}{c}\text { Objets d'observation } \\
\text { Discipline }\end{array}$} & \multicolumn{3}{|c|}{$\begin{array}{l}\text { Enseignant } \\
\text { compétent }\end{array}$} & \multicolumn{3}{|c|}{ Enseignant efficace } & \multicolumn{3}{|c|}{ Enseignant expert } \\
\hline & Math & $\mathbf{P C}$ & SVT & $\begin{array}{c}\text { Mat } \\
\text { h }\end{array}$ & $\mathbf{P C}$ & SVT & $\begin{array}{c}\text { Mat } \\
\text { h }\end{array}$ & PC & SVT \\
\hline \multicolumn{10}{|l|}{ Connaissances de la discipline } \\
\hline - Épistémologie & & & & & & & & & \\
\hline$\circ$ Pertinence des démarches & + & + & + & + & + & + & + & + & + \\
\hline $\begin{array}{l}\text { - Pertinence des méthodes utilisées } \\
\text { (induction, déduction, généralisation) }\end{array}$ & $+/-$ & $+/-$ & $+/-$ & $+/-$ & $+/-$ & $+/-$ & + & + & + \\
\hline - Pertinence des types connaissances & $+/-$ & $+/-$ & $+/-$ & $+/-$ & $+/-$ & $+/-$ & + & + & + \\
\hline $\begin{array}{l}\text { - Cohérence dans leur utilisation } \\
\text { - Pertinence des preuves avancées }\end{array}$ & $+/-$ & $+/-$ & $+/-$ & $+/-$ & $+/-$ & $+/-$ & $+/-$ & $+/-$ & $+/-$ \\
\hline \multicolumn{10}{|l|}{ Histoire des concepts } \\
\hline$\circ$ Origine logique & $+/-$ & $+/-$ & $+/-$ & $+/-$ & $+/-$ & $+/-$ & $+/-$ & $+/-$ & $+/-$ \\
\hline ○ Leur valeur & $+/-$ & $+/-$ & $+/-$ & $+/-$ & $+/-$ & $+/-$ & $+/-$ & $+/-$ & $+/-$ \\
\hline Leur portée objective & $+/-$ & $+/-$ & $+/-$ & $+/-$ & $+/-$ & $+/-$ & $+/-$ & $+/-$ & $+/-$ \\
\hline \multicolumn{10}{|l|}{ - Approche interdisciplinaire } \\
\hline $\begin{array}{l}\text { Rapport interne entre les disciplines } \\
\text { du domaine }\end{array}$ & $+/-$ & $+/-$ & $+/-$ & $+/-$ & $+/-$ & $+/-$ & $+/-$ & $+/-$ & $+/-$ \\
\hline $\begin{array}{l}\text { Interrelations entre les théories des } \\
\text { différentes disciplines du domaine }\end{array}$ & $+/-$ & $+/-$ & $+/-$ & $+/-$ & $+/-$ & $+/-$ & $+/-$ & $+/-$ & $+/-$ \\
\hline
\end{tabular}


Nous notons que les enseignants réussissent bien aux niveaux de la Pertinence des démarches et de la Pertinence des méthodes utilisées, deux des cinq composantes de l'Epistémologie. L'analyse montre l'existence des difficultés aussi bien aux niveaux de la Pertinence des types de connaissances, de la Cohérence dans leur utilisation, et de la Pertinence des preuves avancées constituant trois des cinq composantes de l'Epistémologie, qu'aux niveaux de ses deux autres composantes des Connaissances de la discipline : Histoire des concepts et Approche interdisciplinaire. Cela résulte du manque de formation initiale des enseignants en épistémologie. Ainsi dans la classe, nous relevons que les enseignants ont peur de s'engager dans un accompagnement de l'élève dans l'exercice de persuasion qu'ils devraient entreprendre. Ces résultats viennent confirmer aussi l'inquiétude de Fourez (1998) qui déplore le fait que l'enseignement des sciences ne soit pas assez lié à l'aspect social des savoirs scientifiques. En effet, ces enseignants en exercice depuis plusieurs années ne sont pas toujours en mesure d'aborder l'aspect social des savoirs scientifiques puisqu'elles n'ont probablement pas été sensibilisées au processus de construction sociale lors de leur formation initiale. Ce qui montre clairement que ces enseignants n'ont pas eu de la formation continue en ce sens. On peut penser que les connaissances produites par les chercheurs ne sont pas enseignées et nécessairement véhiculées en milieu de pratique. Les savoirs à leurs complexes de transformations aux différentes étapes de sélection et de leur enseignement, qui impliquent des connaissances fondamentalement divergentes des savoirs académiques. Pour ces enseignants, nous notons que l'enseignement d'une matière leur pose un défi de taille sur le plan de la compréhension des concepts, supposant ainsi que son enseignement requiert une aisance que certains n'ont pas. Cette situation s'explique par la formation universitaire qu'ils ont reçue, la majorité de ces derniers était formée dans leur discipline de base. Cela dépeint du moins en partie le lourd travail d'appropriation de contenus notionnels de certains enseignants de sciences lorsque que l'enseignement de leur discipline leur est confié (Fourez et al., 1997 ) ; ils ne sont pas formés non plus pour poser un regard épistémologique sur leurs prises de position didactiques. Ainsi, l'approche interdisciplinaire ou l'intégration horizontale reliant les connaissances et les habiletés interdépendantes de plus d'une matière pour en étudier un thème central, un enjeu, un problème, un sujet ou une expérience devient problématique. Il est donc clair que les enseignants manquent d'outils pour être plus critiques à leur pratique.

\section{- Troisième objet d'observation : les connaissances curriculaires}

Ensemble des pratiques éducatives relatives à la maîtrise des programmes éducatifs que l'enseignant doit non seulement connaitre, tant du point de vue des concepts à enseigner que des compétences exigibles, mais également les 
choix axiologiques des concepteurs relatifs aux finalités de l'enseignement de la discipline et à ses modalités d'approche. Ils interprètent les différents programmes en fonction du temps disponible, des caractéristiques des élèves et de différentes exigences du contexte. D'emblée, les enseignants soulignent l'importance d'une connaissance globale des programmes, c'est-à-dire de leurs contenus, de leurs différents objectifs, selon l'année d'enseignement concernée. Cependant, il ne suffit pas d'en connaître les contenus. Il faut savoir discriminer ce qui est essentiel de ce qui ne l'est pas. Il faut reconnaître parmi l'ensemble des objectifs quels sont les éléments importants, lesquels sont désignés par la mention « objectif terminal » (Méheut, 2006). Le tableau 5 répertorie les évaluations relevées des observations de classe des trois profils d'enseignants au regard des connaissances de la discipline. Elle constitue l'architecture pédagogique d'un système éducatif, et conditionne à la fois la manière dont sont rédigés les programmes d'études, les pratiques de classe, l'évaluation des acquis des élèves, la formation des enseignants et la conception des manuels scolaires. 
Tableau 5 : synthèse des observations au regard des connaissances curriculaires pour les trois profils d'enseignants choisis

\begin{tabular}{|c|c|c|c|c|c|c|c|c|c|c|}
\hline \multirow{2}{*}{\multicolumn{2}{|c|}{$\begin{array}{c}\text { Objets d'observation } \\
\text { Discipline }\end{array}$}} & \multicolumn{3}{|c|}{ Enseignant compétent } & \multicolumn{3}{|c|}{ Enseignant efficace } & \multicolumn{3}{|c|}{ Enseignant expert } \\
\hline & & Math & $\mathbf{P C}$ & SVT & Math & PC & SVT & Math & PC & SVT \\
\hline \multicolumn{11}{|c|}{ Situations d'apprentissage } \\
\hline & $\circ$ Maîtrise des types de texte & $+/-$ & $+/-$ & $+/-$ & $+/-$ & $+/-$ & $+/-$ & $+/-$ & $+/-$ & $+/-$ \\
\hline & ○ Maîtrise des styles pédagogiques & $+/-$ & $+/-$ & $+/-$ & + & + & + & + & + & + \\
\hline- & Référence à la dimension sociale et culturelle & - & - & - & - & - & - & - & - & - \\
\hline & Référence à la vulgarisation scientifique & - & - & - & - & - & - & - & - & - \\
\hline- & Maîtrise des activités et leur durée & $+/-$ & $+/-$ & $+/-$ & $+/-$ & $+/-$ & $+/-$ & $+/-$ & $+/-$ & $+/-$ \\
\hline
\end{tabular}


Les résultats obtenus en rapport avec les Connaissances curriculaires montrent que les enseignants observés ont une maîtrise acceptable de ces connaissances. Il s'agit de la maîtrise des styles pédagogiques, des savoirs à mobiliser, et des compétences à installer. Cependant, nous relevons que ces enseignants ne font pas référence dans leur enseignement à la dimension sociale et culturelle. Ces résultats viennent confirmer aussi l'inquiétude de Fourez (1998) qui déplore le fait que l'enseignement des sciences ne soit pas assez lié à l'aspect social des savoirs scientifiques. En effet, ces enseignants en exercice depuis plusieurs années ne sont pas toujours en mesure d'aborder l'aspect social des savoirs scientifiques puisqu'elles n'ont probablement pas été sensibilisées au processus de construction des connaissances puisqu'ils n'ont pas eu de la formation continue en ce sens. On note aussi que les connaissances produites par les chercheurs ne sont pas prises en compte dans leur enseignement. Or se référer à la vulgarisation scientifique pour rendre compréhensible son enseignement permet à l'apprenant de se situer dans le monde afin de sociale lors de leur formation initiale. Ce qui montre clairement que guider sa vie, il est évident que la connaissance de la pensée scientifique et de ses conséquences sociales est indispensable à l'homme cultivé (Jeanneret, 1994) d'autant qu'elle apporte non seulement des connaissances mais aussi contribue à créer dans la compréhension des phénomènes des attitudes nouvelles, un sens critique plus aigu, et une objectivité plus grande.

\section{- Quatrième objet d'observation : les connaissances pédagogiques}

Les connaissances pédagogiques sont les connaissances, des stratégies d'enseignement tant aux niveaux de la planification, de la motivation des apprenants que de la gestion de la classe. Elles font référence aux connaissances générales de l'enseignant à propos de l'enseignement, sans être liées à un contenu disciplinaire. Par exemple on peut citer les connaissances mises en œuvre par l'enseignant lorsque celui-ci désire ramener le calme dans la classe. Les connaissances pédagogiques liées au contenu sont les connaissances que développe un enseignant pour aider les élèves à comprendre et apprendre un contenu (Shulman, 1987). Elles sont relatives à l'organisation de la leçon, au choix des activités à mettre en place pour faciliter la compréhension des concepts par les apprenants, à l'organisation et à la gestion des interactions dans la classe pour permettre aux élèves de raisonner et de donner du sens aux notions introduites. Le tableau 6 répertorie les évaluations relevées sur les observations de classe des trois profils d'enseignants au regard des connaissances pédagogiques. 
Tableau 6 : synthèse des observations au regard des connaissances pédagogiques pour les trois profils d'enseignants choisis

\begin{tabular}{|c|c|c|c|c|c|c|c|c|c|c|}
\hline \multirow{2}{*}{\multicolumn{2}{|c|}{$\begin{array}{c}\text { Objets d'observation } \\
\text { Discipline }\end{array}$}} & \multicolumn{3}{|c|}{ Enseignant compétent } & \multicolumn{3}{|c|}{ Enseignant efficace } & \multicolumn{3}{|c|}{ Enseignant expert } \\
\hline & & Math & PC & SVT & Math & $\mathbf{P C}$ & SVT & Math & PC & SVT \\
\hline \multicolumn{11}{|c|}{ Connaissances pédagogiques } \\
\hline \multirow[t]{11}{*}{-} & Les stratégies d'enseignement & & & & & & & & & \\
\hline & o Présenter clairement les contenus & & & & & & & & & \\
\hline & d'apprentissage aux élèves & $+/-$ & $+/-$ & $+/-$ & + & + & + & + & + & + \\
\hline & $\begin{array}{l}\text { Offrir un enseignement } \\
\text { supplémentaire }\end{array}$ & - & - & - & - & - & - & - & - & - \\
\hline & ○ Favoriser l'engagement des élèves & & & & & & & & & \\
\hline & envers les contenus d'apprentissage & + & + & + & + & + & + & + & + & + \\
\hline & ○ Donner des rétroactions & + & + & + & + & + & + & + & + & + \\
\hline & $\circ$ Miser sur la pratique & $+/-$ & $+/-$ & $+/-$ & $+/-$ & $+/-$ & $+/-$ & + & + & + \\
\hline & $\begin{array}{l}\text { Aider les élèves à utiliser leurs } \\
\text { connaissances }\end{array}$ & $+/-$ & $+/-$ & $+/-$ & $+/-$ & $+/-$ & $+/-$ & $+/-$ & $+/-$ & $+/-$ \\
\hline & ○ Faire travailler les élèves ensemble & + & + & + & + & + & + & + & + & + \\
\hline & ○ Développer l'auto-efficacité des élèves & - & - & - & - & - & - & - & - & - \\
\hline \multicolumn{11}{|c|}{ 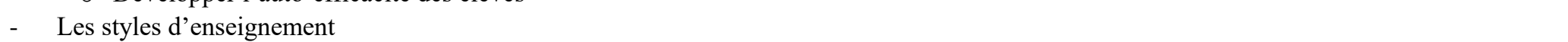 } \\
\hline & $\circ$ Style transmissif & + & + & + & + & + & + & + & + & + \\
\hline & $\circ$ Style incitatif & $+/-$ & $+/-$ & $+/-$ & $+/-$ & $+/-$ & $+/-$ & $+/-$ & $+/-$ & $+/-$ \\
\hline & - Style associatif & + & + & + & + & + & + & + & + & + \\
\hline & $\circ$ Style permissif & $+/-$ & $+/-$ & $+/-$ & $+/-$ & $+/-$ & $+/-$ & $+/-$ & $+/-$ & $+/-$ \\
\hline \multicolumn{11}{|c|}{ - $\quad$ Les types de questions } \\
\hline & - Vrai ou Faux & $+/-$ & $+/-$ & $+/-$ & $+/-$ & $+/-$ & $+/-$ & $+/-$ & $+/-$ & $+/-$ \\
\hline & ○ Fermées & + & + & + & + & + & + & + & + & + \\
\hline & - Ouvertes & $+/-$ & $+/-$ & $+/-$ & $+/-$ & $+/-$ & $+/-$ & $+/-$ & $+/-$ & $+/-$ \\
\hline
\end{tabular}


Les résultats obtenus en rapport avec les Connaissances pédagogiques montrent que les enseignants observés ont des difficultés dans la maîtrise des composantes des styles d'enseignement, et des types de questions. Leur incertitude face aux composantes ci-dessus mentionnées et aux pratiques pédagogiques les amène à privilégier les propositions didactiques qu'elles transposent à nouveau au gré de leurs compétences notionnelles et didactiques (Carette, 2008). C'est ainsi que ces enseignants jouent un rôle plutôt technique par rapport aux démarches qu'ils utilisent et n'entrent pas dans un processus d'accompagnement des apprenants au moment de l'exercice de persuasion pourtant nécessaire à la compréhension. Ces difficultés constatées sont dues au fait que ces enseignants ne sont pas formés non plus pour poser un regard épistémologique sur leurs prises de position didactiques (Fourez et al., 1997). En somme, ces résultats mènent à des pistes pour la formation initiale et également pour la formation continue. Puisque l'obtention d'un diplôme passe par l'acquisition de connaissances et la réussite dans certaines matières scolaires, la façon de transmettre ces connaissances joue aussi un rôle dans la persévérance scolaire des jeunes. Les pratiques pédagogiques et éducatives de l'enseignant réalisées les plus efficacement possibles auront un effet sur l'intérêt général de l'élève pour la matière enseignée, et plus largement, sur son appréciation de l'expérience de l'apprentissage. À ce titre, les stratégies pédagogiques gagnantes relèvent autant de la gestion de classe que des techniques d'enseignement employées (Cusset, 2011). Pour avoir l'adhésion des apprenants, les enseignants doivent tout d'abord acquérir ces connaissances, et développer de nouvelles pratiques pédagogiques favorisant la construction de connaissances. Ils doivent ensuite apprendre à observer de nouveaux indicateurs dans le processus d'apprentissage des élèves et maitriser un bagage d'exemples et de contrexemples permettant de saisir les occasions d'apprentissage et, enfin, percevoir l'effet de ces changements sur le processus d'apprentissage et la compétence à écrire des apprenants. « Il va sans dire que toutes les pratiques pédagogiques ne sont pas égales [...]. Il importe par conséquent d'identifier et de promouvoir les pratiques les plus efficaces, c'està-dire celles qui aident les élèves à apprentissages souhaités. » (Gauthier et al., 2004). Il est donc nécessaire de repenser la formation des enseignants, leur accompagnement et développement professionnel, basé sur une bonne connaissance de la recherche.

\section{- Cinquième objet d'observation : les connaissances didactiques}

Les connaissances didactiques sont les difficultés d'apprentissage que sont susceptibles de rencontrer les apprenants et la manière de les prendre en compte. Pour Morge (2009), elles ont la particularité d'être dépendantes du contenu disciplinaire enseigné ce qui en fait des connaissances beaucoup plus précises que des connaissances sur la discipline scolaire, et que certaines ne 
sont même utilisables que pour une séance donnée. Lebrun et De Hosson (2017) indiquent qu'elles sont «largement dépendantes des contenus disciplinaires à enseigner» et correspondent aux «connaissances que l'enseignant a de ce qui doit être enseigné, de la difficulté ou au contraire du caractère simple d'un enseignement, des difficultés possibles des élèves (audelà d'une connaissance de leurs acquis) ». Les connaissances didactiques sont donc liées à l'action, et peuvent s'acquérir ou être modifiées par la pratique professionnelle. Le tableau 7 répertorie les évaluations relevées des observations de classe des trois profils d'enseignants au regard des connaissances pédagogiques. 
Tableau 7 : synthèse des observations au regard des connaissances didactiques pour les trois profils d'enseignants choisis

\begin{tabular}{|c|c|c|c|c|c|c|c|c|c|c|}
\hline \multirow{2}{*}{\multicolumn{2}{|c|}{$\begin{array}{c}\text { Objets d'observation } \\
\text { Discipline }\end{array}$}} & \multicolumn{3}{|c|}{ Enseignant compétent } & \multicolumn{3}{|c|}{ Enseignant efficace } & \multicolumn{3}{|c|}{ Enseignant expert } \\
\hline & & Math & PC & SVT & Math & PC & SVT & Math & PC & SVT \\
\hline \multicolumn{11}{|c|}{ Connaissances didactiques } \\
\hline \multicolumn{11}{|c|}{ I ransposition didactique } \\
\hline & ○ Externe & $+/-$ & $+/-$ & - & $+/-$ & $+/-$ & $+/-$ & $+/-$ & $+/-$ & $+/-$ \\
\hline & $\circ$ Interne & + & + & + & + & + & + & + & + & + \\
\hline- & Représentations & $+/-$ & $+/-$ & $+/-$ & $+/-$ & $+/-$ & $+/-$ & $+/-$ & $+/-$ & $+/-$ \\
\hline- & Obstacles & $+/-$ & $+/-$ & $+/-$ & $+/-$ & $+/-$ & $+/-$ & $+/-$ & $+/-$ & $+/-$ \\
\hline- & Niveaux de formulation & + & + & + & + & + & + & + & + & + \\
\hline- & Contrat didactique & + & + & + & + & + & + & + & + & + \\
\hline
\end{tabular}


L'analyse du tableau 7 relève que les enseignants ne connaissent pas de grandes difficultés aux niveaux de la transposition didactique, et les niveaux de formulation. Notons cependant qu'aux niveaux des représentations et les obstacles, les enseignants éprouvent d'énormes difficultés. Cela indique qu'ils n'ont pas été formés de façon formelle aux concepts didactiques. Ce qui confirme d'ailleurs les propos de Jonnaert (1995) selon lesquels la formation didactique devrait non seulement fournir des outils pédagogiques et didactiques aux enseignants, mais aussi les amener à être plus critiques face à leur pratique. Plusieurs didacticiens insistent sur l'importance d'explorer pour chaque concept à enseigner les conceptions prégnantes et récurrentes qui se rencontrent chez les élèves car elles sont résistantes aux efforts d'enseignement. Cette résistance est le résultat de la présence d'un petit nombre d'obstacles d'ordre épistémologique et/ou imaginaire munis de structures fortement ancrées et fonctionnelles chez l'apprenant. Astolfi (2001) qualifie cette résistance de rationnelle puisqu'elle est la manifestation d'économies de pensées, d'une bonne forme de perception stable à laquelle l'esprit de l'élève fait continuellement référence. Les obstacles constituent alors le noyau dur des représentations, ils leur procurent un sens ce qui permet d'en dégager une interprétation. L'évolution des connaissances en didactique des sciences concernant les représentations et les obstacles a également favorisé la considération du conflit cognitif. Ce conflit a été défini par Piaget (1975) comme la possibilité qu'un même sujet dispose de systèmes différents selon les domaines et les situations. D'autres chercheurs (Joshua \& Dupin, 1989 ; 1993) ont repris ces travaux pour leur attribuer une dimension plus sociale d'où l'expression conflit sociocognitif.

\section{- Sixième objet d'observation : les connaissances des TICE}

Les TICE représentent les Technologies de l'Information et de la Communication pour l'Enseignement. Elles recouvrent les outils et produits numériques pouvant être utilisés dans le cadre de l'éducation et de l'enseignement $(\mathrm{TICE}=\mathrm{TIC}+$ Enseignement). Les TICE regroupent un ensemble d'outils conçus et utilisés pour produire, traiter, entreposer, échanger, classer, retrouver et lire des documents numériques à des fins d'enseignement et d'apprentissage. En clair, il s'agit de mettre en avant les technologies modernes pour les inclure dans l'enseignement. On peut aussi parler de l'utilisation en contexte pédagogique des outils numériques (outils TICES) au service des stratégies d'apprentissage. Une des questions essentielles aux enseignants, c'est comment intégrer les TICE dans sa pédagogie ? Le tableau 8 répertorie les évaluations relevées sur les observations de classe des trois profils d'enseignants au regard des connaissances des TICE. 
Tableau 8 : synthèse des observations au regard des connaissances des TICE pour les trois profils d'enseignants choisis

Objets d'observation Discipline

Connaissances des TICE

Connaissances des TICE

- Pertinence des documents utilisés

Enseignant compétent

Math PC SVT

PC

$+/-\quad+/-\quad+/-$

$+/-\quad+/-\quad+/-$

Enseignant efficace

Math PC SVT

Enseignant expert

Math

$\begin{array}{lll}+/- & +/- & +/- \\ +/- & +/- & +/-\end{array}$

$+/-$

PC SVT

$+$

$+/-$


L'observation des pratiques intégrant les TICE dans le tableau 8 montre de prime abord une grande difficulté dans l'utilisation des outils informatiques et dans la pertinence des documents utilisés. Ce constat général reste inchangé lorsqu'il se décline dans différentes situations. Il s'agit d'un manque d'appétence des enseignants. En effet, les TICE fournissent des moyens novateurs, non seulement pour la diffusion des connaissances mais aussi pour l'exploration de stratégies d'apprentissage qui favorisent la construction des compétences (Lebrun, 2002). ). Le « contexte » large de l'Education nationale montre une volonté forte d'intégration et d'utilisation des TICE à l'école et dans les pratiques pédagogiques. Ces enseignants n'y font pas référence et se focalisent plutôt sur le contexte proche. Ils y trouvent des arguments en défaveur de l'utilisation des TICE, notamment, l'absence de matériel directement disponible dans la classe, le manque de compétences techniques et l'insuffisance, en quantité et qualité, de l'offre de la formation continue en matière d'usages de ces technologies dans l'enseignement. D'ailleurs, comme le précise Poellhuber et Boulanger (2001), ce n'est pas l'utilisation de la technologie en soi qui importe, ce sont les usages qu'on en fait. C'est donc à une pédagogie active que nous invitent les TIC, à une pédagogie mettant davantage l'accent sur l'activité d'apprentissage des élèves que sur l'activité d'enseignement de l'enseignant.

\section{Conclusion:}

Il est étonnant de constater après cette étude que les trois types d'enseignants observés et interviewés montrent tant de lacunes dans la maîtrise des six objets d'observation: situations d'apprentissage, connaissances de la discipline, connaissances curriculaires, connaissances pédagogiques, connaissances didactiques et connaissances des TICE.

Les résultats des analyses indiquent que la mauvaise lecture des intentions didactiques des programmes éducatifs par les enseignant(e)s amène ces derniers à adopter des pratiques d'enseignement qui favorisent le décrochage scolaire, entraînant ainsi la montée des inégalités dans l'obtention des diplômes scolaires.

Par conséquent, certaines stratégies d'enseignement en fonction du contexte scolaire auquel l'enseignant(e) fait face sont à ajuster dans leurs aspects didactique et pédagogique pour donner aux élèves l'envie de suivre, de poursuivre les études, de développer plus de compétences, et réduire conséquemment les inégalités scolaires.

Notre propos a amené des clarifications sur les représentations de l'enseignement des sciences et sur les pratiques pédagogiques déployées par trois types d'enseignants régulièrement affectés aux élèves des classes de $3^{\text {ème }}$ ( $4^{\text {ème }}$ année de collège). Ces clarifications ont une portée limitée car le nombre de sujets est restreint et nous n'avons pu avoir accès à un nombre élevé 
d'enseignants concernés. Nous relevons cependant que leur formation initiale ne les habilite pas adéquatement à l'enseignement des disciplines du domaine « sciences », et ce, tant au plan notionnel qu'au plan pédagogique. Une réflexion en ce sens est une piste à explorer si on veut qu'elles entreprennent un véritable changement pédagogique. Cette réflexion les aiderait à comprendre la complexité du processus de construction des savoirs et à faire des choix pédagogiques éclairés et pertinents.

\section{References:}

1. Abell, S.K. (2007). Research on Science Teacher Knowledge. In S.K. Abell and N.G. Lederman (Eds) Handbook of research on Science Education (pp 1105 - 1149). New Jersey: Lawrence Erlbaum Associates Inc.

2. Astolfi, J.-P. (2001). L'erreur, un outil pour enseigner, ESF Editeur.

3. Carette, V. (2008). «Les caractéristiques des enseignants efficaces en question ». Revue française de pédagogie, $n^{\circ} 162$, p. 81-93.

4. Cusset, P.-Y. (2011). « Que disent les recherches sur "l'effet enseignant»? ». Note d'analyse, $n^{\circ} 232$, p. 1-12.

5. Coleman, J. S., Campbell, E. Q., Hobson, C. J., McPartland, J., Mood, A. M. \& Weinfeld, F. D. (1966). Equality of Educational Opportunity. Washington, U.S. Department of Health, Education and Welfare.

6. Crahay, M., Marbaise, C. \& Issaieva, E. (2013). What is teachers' belief in the virtues of student retention founded on ? Giornale Italiano della Ricerca Educativa - Italian Journal of Educational Research, 11, pp.75-94.

7. Foulezis, G. (2012). Les enquêtes PISA Avec Samuel Charmillot Coll. Que sais-je ? (Presses Universitaires de France).

8. Felouzis, G., B. Fouquet-Chauprade, S. Charmillot, et L. ImperialeArefaine (2016). Inégalités scolaires et politiques d'éducation. Contribution au rapport du Cnesco : Comment l'école amplifie-t-elle les inégalités sociales et migratoires ?

9. Fourez, G. (1998). Se représenter et mettre en œuvre l'interdisciplinarité à l'école. Revue des sciences de l'éducation, XXIV (1), 31-50.

10. Fourez, G., Englebert-Lecomte, V. et Mathy, P. (1997). Nos savoirs sur nos savoirs - Un lexique d'épistémologie pour l'enseignement. Bruxelles : De Bœck Université.

11. Gauthier, C. \& Dembélé, M. (2004). Qualité de l'enseignement et qualité de l'éducation : Revue des résultats de recherche. Paris : UNESCO, n 2005/ED/ EFA/MRT/PI/18. 
12. Gueudet, G. et Trouche, L. (2010). Ressources vives : le travail documentaire des professeurs en mathématiques. Rennes, France : Presses universitaires de Rennes.

13. Grossman, P.L. (1990). The making of a teacher : teacher knowledge and teacher education. New York : Teachers College Press.

14. Jeanneret, Y. (1994). Écrire la science, Formes et enjeux de la vulgarisation, Paris, Presses Universitaires de France, 398 p.

15. Jencks, C. (1992). Rethinking Social Policy: Race Poverty and the Underclass, Havard University Press.

16. Jonnaret, Ph. (1995). Entrer dans l'apprentissage scolaire. In G. Forges, (éd.), Enfants issus de l'immigration et apprentissage du français langue seconde, pp. 15-53. Paris, FR : Didier-Erudition.

17. Jonnaert, Ph. (2007). Le constructivisme comme fondement des réformes contemporaines des systèmes éducatifs. Dakar : Éditions des écoles nouvelles africaines - Sénégal.

18. Jonnaert, Ph.; Barrette, J.; Boufrahi, S.; Masciotra, D. (2005). Contribution critique au développement des programmes d'études: compétences, constructivisme et interdisciplinarité. Revista de Ciencias de la Educación, 3, 667-696.

19. Jonnaert, Ph. y Masciotra, D. (dir.) (2004). Constructivisme et choix contemporains en éducation: Hommage à Ernst von Glasersfeld. SteFoy: sa de la Universidad de Quebec.

20. Johsua, S. et Dupin, J.- J. (1989). Représentations et modélisations. Le "débat scientifique " dans la classe d'enseignement de physique. Peter Land. Berne.

21. Johsua, S. et Dupin, J.- J. (1993). Introduction à la didactique des sciences et des mathématiques. Paris : PUF, 422 p. - (Coll. 1er cycle).

22. Lebrun, M. (2002). Des technologies pour enseigner et apprendre. Paris, De Boeck, 2éme édition

23. Lebrun, N. \& de Hosson, C. (2017). Repérer des conceptions d'étudiants : un pas vers l'enrichissement des connaissances professionnelles didactiques d'enseignants-chercheurs de physique. Recherches en didactique des sciences et des technologies, $15,59-96$.

24. Magnusson, S., Krajcik, J., \& Borko, H. (1999). Nature, sources and development of pedagogical content knowledge for science teaching. Dans J. Gess-Newsome \& N. Lederman (dir.), Examining pedagogical content knowledge: The construct and its implications for science education (p. 95-132). Boston : Kluwer.

25. Masciotra, D. et Medzo, F. (2009). Développer un agir compétent. Vers un curriculum pour la vie. Perspectives en éducation et formation. De Boeck Supérieur. 
26. Méheut, M. (2006). Recherches en didactique et formation des enseignants de sciences. In Commission européenne. Direction générale de l'éducation et de la culture (éd.), L'enseignement des sciences dans les établissements scolaires en Europe. États des lieux des politiques et de la recherche. Bruxelles, Eurydice, p. 55-76.

27. Ministère de l'Education nationale (2011). Rapport d'Etat sur le Système Educatif National. Côte d'Ivoire.

28. Morge, L. (2009). La simulation croisée pour accéder aux connaissances professionnelles didactiques locales (LPCK) acquises par l'expérience. In Sixièmes journées scientifiques de l'ARDiST Nantes, 14-16 octobre 2009.

Enligne : http://www.ardist.org/wpcontent/textes\%20communications 24_Morge\%20L.pdf

29. Otis, F. et Ouellet, L. (1996). Le profil de sortie, étape préliminaire à l'élaboration de l'épreuve synthèse de programme, Pédagogie collégiale, 10(1), 9-13.

30. Piaget, J. (1975). A teoria de Piaget. In: MUSSEN, P. H. (org). Psicologia da criança. Desenvolvimento Cognitivo. São Paulo : E.P.U. Vol. 4, p. 71-117.

31. Poellhuber, B., \& Boulanger, R. (2001). Un modèle constructivisme d'intégration des TIC. Rapport PAREA, Trois-Rivières, Collège La flèche, $204 p$.

32. Rochex J.-Y., (2008). " Vingt-cinq ans de politique d'éducation prioritaire en France : une spécificité incertaine et des résultats décevants ", in Demeuseet al. (dir.)Les Politiques d'éducation prioritaire en Europe, INRP.

33. Roegiers, X. (2007). "Curricular reforms guide schools: but, Where to?" Prospects 142(2) : 155-186.

34. Scallon, G. (2004). L'évaluation des apprentissages dans une approche par compétences. Bruxelles: De Boeck.

35. Shulman, L. S. (1986). Those who understand: Knowledge growth in teaching. Educational researcher, 15(2), 4-14.

36. Shulman, L. S. (1987). Knowledge and Teaching_Foundations of the New Reform. Harvard Educational Review, 57(1), 1-23.

37. UNESCO (2013). Document de présentation de l'état des lieux. Renforcement des capacités des encadreurs pédagogiques et formation continue des enseignants. Projet UNESCO Fonds Chinois en dépôt. Côte d'Ivoire. http://unesdoc.unesco.org/images/0022/002298/229860F.pdf

38. Vergnaud, G. (1996). Au fond de l'action, la conceptualisation. Dans J. Bardier (dir.), Savoirs didactiques et savoirs d'action. Paris: PUF 
39. Wang, M., Haertel, G. et Walberg, H. (1990). "What Influences Learning? A Content Analysis of Review Literature", Journal of Educational Research, $\mathrm{n}^{\circ}$ 84, p. 30-43. 